NOT FOR QUOTATION WITHOUT PERMISSION

OF THE AUTHOR

HEAVY VIABLE TRAJECTORIES

OF CONTROLLED SYSTEMS

Jean-Pierre Aubin

Halina Frankowska

April 1984

WP $-84-28$

Working Papers are interim reports on work of the International Institute for Applied Systems Analysis and have received only limited review. Views or opinions expressed herein do not necessarily represent those of the Institute or of its National Member Organizations.

INTERNATIONAL INSTITUTE FOR APPLIED SYSTEMS ANALYSIS A-2361 Laxenburg, Austria 
PREFACE

In this paper the autbors define and study heavy viable trajectories of a controlled system with feedbacks. Viable trajectories of a controlled system are those which satisfy, at each instant, given constraints on the state. The controls regulating viable trajectories evolve according to a set-valued map. Heavy viable trajectories are those which are associated with the controls in the feedback map which have a velocity, at each instant, of minimal norm. The differential equation governing the evolution of the controls associated with heavy viable trajectories is constructed.

This research was conducted within the framework of the Dynamics of Macrosystems study in the System and Decision Sciences Program.

ANDRZEJ WIERZBICKI

Chairman

System and Decision Sciences Program 
ABSTRACT

We define and study the concept of heavy viable trajectories of a controlled system with feedbacks. Viable trajectories are trajectories satisfying at each instant given constraints on the state. The controls regulating viable trajectories evolve according a set-valued feedback map. Heavy viable trajectories are the ones which are associated to the controls in the feedback map whose velocity has at each instant the minimal norm. We construct the differential equation. governing the evolution of the controls associated to heavy viable trajectories and we prove their existence. 
HEAVY VIABLE TRAJECTORIES OF CONTROLLED SYSTEMS

Jean-Pierre Aubin and Halina Frankowska

CEREMADE, Universite de Paris-Dauphine, 75775 Par1s CX (16), France

1. Introduction

When we study the evolution of macrosystems which arise in economics and the social sciences as well as in biological evolution, we should take into account not only :

(1) our ignorance of the future environment of the system but also :

(2) the absence of determinism (including the impossibility of a comprehensive description of the dynamics of the system)

(3) our ignorance of the laws relating certain controls to the states of this system

(4) the variety of dynamics available to the system.

We propose to translate these requirements into mathematics by means of differential inclusions, which describe how the velocity depends in a multi-valued way upon the current state of the system. Another feature of such macrosystems is that the state of the system must obey given restrictions known as viability constraints, which determine the viability domain ; viable trajectories are those lying entirely within the viability domain. Finding viable trajectories of a differential inclusion provides a mechanism of selection of trajectories which, contrary to optimal control theory, does not assume implicitely

(1) the existence of a decision maker operating the controls of the system (there may be more than one decisionmaker in a game-theoretical setting)

(2) the availability of information (deterministic or stochastic) on the future of the system; this is necessary to define the costs associated with the trajectories

(3) that decisions (even if they are conditional) are taken once and for all at the initial time. 
Viability Theorems provide necessary and sufficient conditions for the existence of at least one viable trajectory starting from any viable initial state. It also provides the feedbacks (concealed in both the dynamics and the viability constraints) which relate the state of the system to the controls. These feedbacks are not necessarily deterministic: they are set-valued maps associating a subset of controls with each state of the system. We observe that the larger these subsets of controls are, the more flexible - and, thus, the more robust - the regulation of the system will be.

Finally the third feature shared by those macrosystems is the high inertia of the controls which change only when the viability of the system is at stake. Associated trajectories are called heavy viable trajectories: they minimize at each instant the norm of the velocity of the control. We shall provide a formal definition of heavy viable trajectories, which requires an adequate concept of derivative of the set-valued feedback map. We show that as long as the state of the system lies in the interior of the viability domain, any regulating control will work. Therefore, along a heavy trajectory, the system can maintain the control inherited from the past. (The regulatory control remains constant even though the state may evolve quite rapidly).

What happens when the state reaches the boundary of the viability domain? If the chosen velocity is "inward" in the sense that it pushes the trajectory back into the domain, then we can still keep the same regulatory control.

However, if the chosen velocity is "outward", we are in a period of crisis and must find, as slowly as possible, another regulatory control such that the new associated velocity pushes the trajectory back into the viability domain.

When this strategy for "structural change" fail, the trajectory "dies" i.e., it is no longer viable (see Figure 1). 


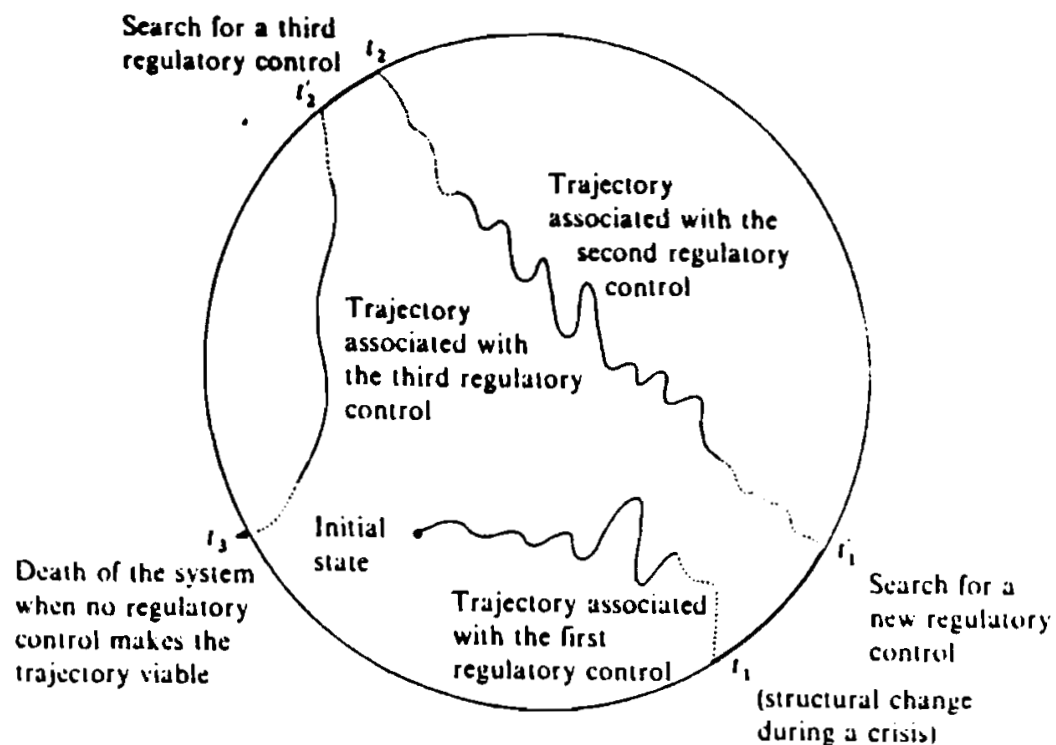

Viability domain

(assumed constant. but which can evolve)

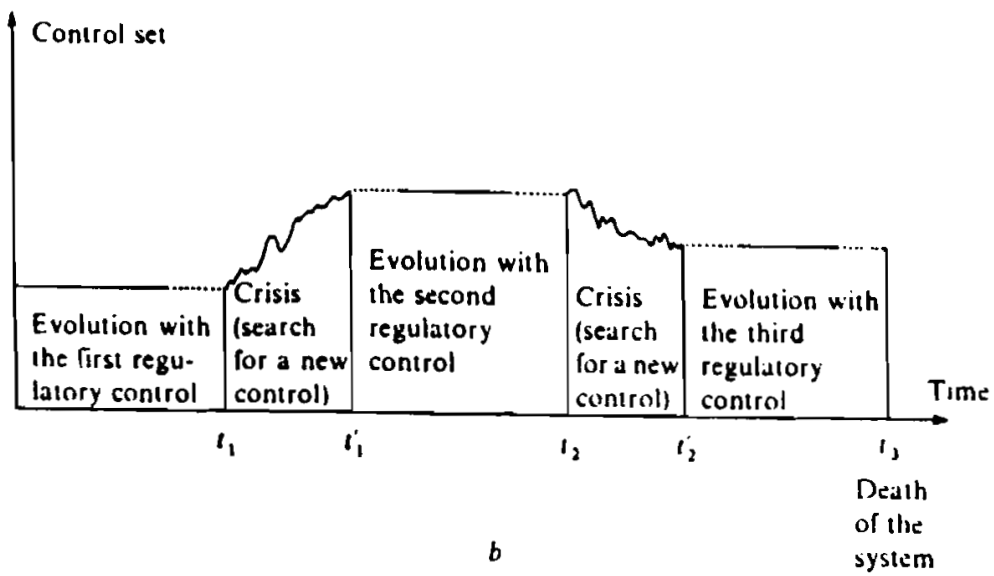

FIGURE 1 (a) Evolution of the state (in the state space) (b) Evolution of the control

The outline of this paper is as follows. In section 2, we define the viability problem for controlled systems with feedbacks, which contain the usual controlled systems and the differential inclusions, and we recall Haddad's viability theorem. We proceed by introducing other tangent cones and by defining contingent derivatives of set-valued maps which we need to define heavy viable trajectories. We define them in section 3 and state the existence theorem of heavy viable trajectories, which we prove in section 4. We give explicit formulas in the smooth case in section 5 . 
The equations which govern the evolution of heavy viable trajectories also reveal a division of the viability domain into "cells" : each cell is the subset of viable states which can be regulated by a given control. To pass from one cell to another requires the control to be changed. The boundaries of these cells signal the need for structural change. We mentionned biological evolution as a motivation for studying heavy viable trajectories. Paleontological concepts such as punctuated equilibria proposed by Elredge and Gould are consistent with the concept of heavy viable trajectories.

Indeed, for the first time, excavations at Kenya's Lake Turkana have provided clear fossil evidence of evolution from one species to another. The rock strata there contain a series of fossils that show every small step of an evolutionary journey that seems to have proceeded in fits and starts. Williamson [1981] examined 3.300 fossils showing how thirteen species of molluscs changed over several million years. What the record indicated was that the animals stayed much the same for immensely long stretches of time. But twice, about 2 million years ago and then again 700.000 years ago, the pool of life seemed to explode - set off, apparently, by a drop in the lake's water level. In an instant of geologic time, as the changing lake environment allowed new types of molluscs to win the race for survival, all of the species evolved into varieties sharply different from their ancestors. That immediate forms appeared so quickly, with new species suddendly evolving in 5.000 to 50.000 years after millions of years of constancy, challenges the traditional theories of Darwin's disciples since the fossils of Lake Turkana don't record any gradual change; rather, they seem to reflect eons of stasis interrupted by brief evolutionary "revolutions". 


\section{$\underline{2 .}$. Background notes.}

We introduce a viability domain $K$, a subset of a finite dimensional space $X$, a finite dimensional control space $U$, a set-valued map $F$ from $K$ to $U$ and a continuous function $f$ from graph(F) to $X$. We define the viability problem for a controlled system with feedbacks as follows: $\forall x_{0} \in K$, find $T>0$ and an absolutely continuous function $x(\cdot)$ satisfying

$$
\left\{\begin{array}{rlll}
\text { i) for almost all } t \in[0, T], & x^{\prime}(t)=f(x(t), u(t)) \\
\text { ii) for almost all } t \in[0, T], & u(t) \in F(x(t)) \\
\text { iii) } & x(0)=x_{0} . &
\end{array}\right.
$$

which are viable on $[0, T]$ in the sense that

$$
\text { for all } t \in[0, T], \quad x(t) \in K \text {. }
$$

By taking $U=X, f(x, u)=u$, we obtain the particular case of a viability problem for a differential inclusion

$$
x^{\prime}(t) \in F(x(t)), \quad x(0)=x_{0}
$$

The viability requirement (2.2) involves naturally restrictions of the dynamical system at the boundary of $K$. It happens that the best way to describe these conditions is to use the contingent cone to $K$ at $x$ (see Aubin-Cellina [1984], p. 176-179, for instance) defined by :

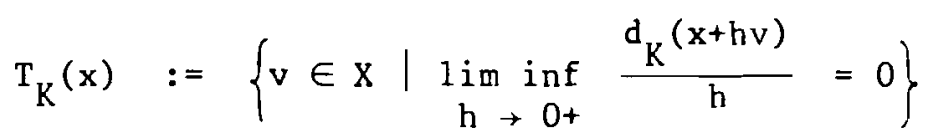

We define the feedback map $R$ from $K$ to $U$ by

$$
R(x) \quad:=\left\{u \in F(x) \mid f(x, u) \in T_{K}(x)\right\}
$$


We observe that any viable trajectory of the controlled system $(2.1)$ is a solution to the "feedback" differential inclusion

$$
\left\{\begin{array}{rlll}
\text { i) for almost all } t \in[0, T], & x^{\prime}(t)=f(x(t), u(t)) \\
\text { ii) for almost all } t \in[0, T], & u(t) \in R(x(t)) \\
\text { iii) } & x(0)=x_{0} &
\end{array}\right.
$$

(the initial set-valued map $F$ is replaced by the feedback map $R$ ),

The main viability theorem (see Haddad [1981], Aubin-Cellina [ 1984 ] p. 239-240) provides necessary and sufficient conditions for the existence of viable trajectories of (2.3).

\section{Theorem 2.1}

We assume that

i) $\mathrm{K}$ is locally compact

ii) $F$ is upper semicontinuous with nonempty convex compact images

iii) $f$ is continuous and is affine with respect to the control

Then the "first order" tangential condition

$$
\forall \mathrm{x} \in \mathrm{K} \quad, \quad \mathrm{R}(\mathrm{x}) \neq \emptyset
$$

is necessary and sufficient for the existence of a viable trajectory of the controlled system (2.1) for all $x_{0} \in K$.

As a by-product of our study of heavy viable trajectories, we shall prove the existence of viable trajectories under another set of assumptions : The convexity of the images of $F$ is no longer required, but we shall need 
the differentiability of $f$ and $F$ (which we shall define) and above all, we need to add to the first-order tangential condition (2.8) a "second order tangential condition" involving the derivative of the feedback map $R$.

Before defining heavy viable trajectories, we need to recall the following facts.

When $K$ is a subset of a finite dimensional $X$, we can define other concepts of tangent cones, among which we mention

a) the tangent cone (introduced by Clarke [1975]):

$$
C_{K}(x):=\left\{v \in X \mid \lim _{\substack{h \rightarrow 0+\\ y \rightarrow x \\ y \in K}} \frac{d_{K}(y+h v)}{h}=0\right\}
$$

b) the Dubovickii-Miljutin [1963] cone :

$$
\mathrm{D}_{\mathrm{K}}(\mathrm{x}):=\{\mathrm{v} \in \mathrm{x} \mid \exists \varepsilon>0 \quad \mathrm{x}+[0, \varepsilon](\mathrm{v}+\varepsilon \mathrm{B}) \subset \mathrm{K}\}
$$

We have the following relations (see Cornet [1981]; Penot [198] ], Aubin-Ekeland [1984] p 409.

and

$$
\mathrm{C}_{\mathrm{K}}(\mathrm{x})=\underset{\substack{\mathrm{y} \rightarrow \mathrm{x} \\ \mathrm{y} \in \mathrm{K}}}{\lim \operatorname{Tinf}_{\mathrm{K}}(\mathrm{y}) \subset \mathrm{T}_{\mathrm{K}}(\mathrm{x})}
$$

$$
\text { Int } C_{K}(x) \subset D_{K}(x) \subset \text { Int } T_{K}(x)
$$

The tangent cone is always convex. It coincides with the contingent cone when $K$ is a smooth manifold (tangent space) or when $K$ is convex or, more generally, when $K$ is soft in the sense that

$$
\mathrm{x} \rightarrow \mathrm{T}_{\mathrm{K}}(\mathrm{x}) \text { is lower semicontinuous. }
$$

(see Aubin-Clarke [1977]). 
Consider now a set-valued map $R$ from $X$ to $U$ and a point $(x, u)$ of its graph. The contingent derivative $D R(x, u)$ is the set-valued map from $X$ to $U$ defined by

$$
w \in D R(x, u)(v) \Leftrightarrow(v, w) \in T_{G r a p h(R)}(x, u)
$$

It is equivalent to say that

$$
\lim _{\substack{h \rightarrow 0+\\ v^{\prime} \rightarrow v}} d\left(w, \frac{R\left(x+h v^{\prime}\right)-u}{h}\right)=0
$$

The contingent derivative $\operatorname{DR}(x, u)$ is a closed process (a map whose graph is a closed cone). We say that the map $F$ is soft if its graph is soft. Then $D R(x, u)$ is a closed convex process, because its graph is equal to the tangent cone to Graph(R) at $(x, u))$. We shall say that $R$ is lower semicontinuously differentiable if

$$
(x, u, v) \rightarrow D R(x, u)(v) \text { is lower semicontinuous }
$$

We observe that in this case $D R(x, u)$ is a closed convex process because property (2.16) implies that $(x, u) \rightarrow T_{G r a p h(R)}(x, u)$ is lower semicontinuous, and thus, Graph $\operatorname{DR}(x, u)$ is a closed convex cone.

Finally, when $K$ is a closed subset of $X$, we denote by

$$
m(K):=\left\{u \in K \mid\|u\|=\min _{v \in K}\|v\|\right\}=\pi_{K}(0)
$$

the subset of elements of $K$ with minimal norm. If $F$ is a continuous set-valued map with closed convex images, the single-valued map $x \rightarrow m(F(x))$ is continuous. This is no longer the case when $F$ is only upper or lower semicontinuous (with closed convex images). However, 


$$
\left\{\begin{array}{l}
\text { if } F \text { is lower semicontinuous with clopsed images, } \\
\text { then } x \rightarrow d(0, F(x)) \text { is upper semicontinuous }
\end{array}\right.
$$

We refer to Aubin [1983] and Aubin-Ekeland, [1984], Chapter 7, Clarke [1983] for a general presentation of nonsmooth analysis releyant to this study.

\section{Heavy viable trajectories.}

We consider the viability problem (2.1), (2.2) for controlled systems with feedbacks. We have seen that viable trajectories are solutions to the feedback differential inclusion (2.6). When the functions $x(\cdot)$ and $u(\cdot)$ are absolutely continuous, we can differentiate the "first order" feedback law

$$
\forall t \in[0, T], \quad u(t) \in R(x(t))
$$

and obtain the "second order" feedback law

$$
\text { for almost all } t \in[0, T], u^{\prime}(t) \in D R(x(t), u(t))(f(x(t), u(t)))
$$

We now propose to select among all regulatory controls satisfying (3.2) the ones whose velocity has a minimal norm : such trajectories seem to be present in the evolution of macrosystems arising in social, economic and biological sciences (which motivated viability theory in the first place).

\section{Definition 3.1}

We shall say that absolutely continuous functions $(x(\cdot), u(\cdot))$ form a heavy viable trajectory if it is a solution to the system of differential inclusions : 


$$
\begin{aligned}
& \text { i) } x^{\prime}=f(x, u) \\
& \text { ii) } u^{\prime} \in m(D R(x, u)(f(x, u))) \\
& \text { iii) }(x(0), u(0)) \text { given in } \operatorname{Graph}(R)
\end{aligned}
$$

which are viable in the sense that

$$
\forall t \in[0, T], \quad x(t) \in K \text { and } u(t) \in R(x(t)) \text {. }
$$

We shall say that the subse:s

$$
C(u) \quad:=\quad\{x \in K \mid 0 \in \operatorname{DR}(x, u)(f(x, u))\}
$$

are the viability cells of the system.

We observe that along a heavy viable trajectory, a system will keep the control $u\left(t_{0}\right)$ as long as the state $x(t)$ remains in the viability cell $C\left(u\left(t_{0}\right)\right)$ for $t \geqslant t_{0}$, because in this case inclusion (3.3)ii) states that $u^{\prime}(t)=0$. If not, when $x(t)$ leaves the viability cell $c\left(u\left(t_{0}\right)\right)$ at time $\bar{t}_{0}$, the control starts to evolve at time $\bar{t}_{0}$ until the time $t_{1}$ when $x\left(t_{1}\right) \in C\left(u\left(t_{1}\right)\right)$.

In the case of ordinary differential inclusions (when $U=X$ and $f(x, u)=u)$, heavy viable trajectories can be written $x\left(t_{0}\right)+\left(t-t_{0}\right) x^{\prime}\left(t_{0}\right)$ when $x\left(t_{0}\right) \in C\left(x^{\prime}\left(t_{0}\right)\right)$ as long as $x\left(t_{0}\right)+\left(t-t_{0}\right) x^{\prime}\left(t_{0}\right)$ remains in $C\left(x^{\prime}\left(t_{0}\right)\right)$. In this case, the viability cells display areas of the viability domain where "linear quantitative growth" holds true.

We observe also the following inclusion

$$
\left\{x \in R^{-1}(u) \mid f(x, u) \in T_{R^{-1}(u)}(x)\right\} \subset C(u)
$$

for all $u \in \operatorname{Im}(R)$. 
We shall state our main existence theorem.

\section{Theorem 3.2}

We assume that $f$ is $C^{1}$ in a neighborhood of Graph(F) and that

$$
\text { the maps } F \text { and } T_{K} \text { are soft }
$$

We posit the following "transversality condition"

$$
\left\{\begin{array}{l}
\forall(x, u) \in \operatorname{Graph}(R), \forall(y, z) \in X x X, \exists v \in X \text { satisfying } \\
z \in f_{x}^{\prime}(x, u) v+f_{u}^{\prime}(x, u) D F(x, u)(v)-D T_{K}(x, f(x, u))(v-y)
\end{array}\right.
$$

Then the derivative of the feedback map $R$ can be written

(3.9) $\left\{\begin{array}{l}D R(x, u)(v)= \\ D F(x, u)(v) \cap f_{u}^{\prime}(x, u)^{-1}\left(D T_{K}(x, f(x, u))(v)-f_{x}^{\prime}(x, u) y\right)\end{array}\right.$

$$
\text { Assume moreover that }
$$

$$
\text { Graph(F) is locally compact }
$$

and that The feedback map $R$ is lower semicontinuously differentiatle

Then the "first order" condition

$$
\forall \mathrm{x} \in \mathrm{K} \quad, \quad \mathrm{R}(\mathrm{x}) \neq \emptyset
$$

and the "second order" condition

$$
\forall(x, u) \in \operatorname{Graph}(R), \quad f(x, u) \in \operatorname{Dom} \operatorname{DF}(x, u) \cap \operatorname{Dom} D_{T}(x, f(x, u))
$$

imply the existence of heavy viable trajectories of the controlled system (2.1) for any initial state $x_{0} \in K$ and initial control $u_{0} \in R\left(x_{0}\right)$ if 
(3.14) Graph $\left(\mathrm{T}_{\mathrm{K}}\right)$ is locally compact

If the graph of $T_{K}$ is not locally compact, the initial control must satisfy

$$
f\left(x_{0}, u_{0}\right) \in D_{K}\left(x_{0}\right)
$$

Several comments are in order. Theorem 3.2 should be compared to Theorem 2.1. Theorem 3.2 does not involve convexity requirements, but smoothness conditions (3.7) and (3.11) and second order condition (3.13) on top of the first order condition (3.12). The solutions are more regular (the control is absolutely continuous instead of being only measurable), but there exist the restriction (3.15) on the initial state when the graph of $\mathrm{T}_{\mathrm{K}}$ is not locally compact. Unfortunately, this happens whenever $K$ involves inequality constraints : Take for instance $K=\mathbf{R}_{+}$. Then $\operatorname{Graph}\left(\mathrm{T}_{\mathrm{K}}\right)=\left(\{0\} \times \mathbf{R}_{+}\right) \cup(] 0, \infty[\times \mathbf{R})$ is not locally compact. But the map $\mathrm{T}_{K}$ is soft and even lower semicontinuously differentiable because

$$
\operatorname{DT}_{K}(x, u)(v)= \begin{cases}\mathbb{R} & \text { if } \quad v \leqslant 0 \\ \emptyset & \text { otherwise }\end{cases}
$$

This crucial example shows that assumption (3.7) is not unreasonable.

Since the maps $F$ and $T_{K}$ are soft, the derivative $D R(x, u)$ defined by (3.) is a closed convex process and the differential inclusicn (3.3)ii) governing the velocity of the control is actually the differential equation

$$
u^{\prime}(t)=m(D R(x(t), u(t))(f(x(t), u(t))))
$$

There are no general explicit formula allowing to couch $m(D R(x, u) f(x, u))$ ) in terms of $D F(x, u)(f(x, u)), D_{K}(x, u)(f(x, u))$ and $f^{\prime}(x, u)$ by using formula (3.9). However, see Annex for some further remarks on this nroblen. 
We can also provide sufficient conditions for the regularity assumption (3.11) to hold true. For instance, thanks to a theorem on the lower semicontinuity of the intersection of two lower semicontinuous maps (see Aubin-Cellina [1984] p. 49), conditions (3.7) and (3.11) follow from the following ones

i) the set-valued maps $F$ and $T_{K}$ are lower semicontinuously differentiable

ii) $(x, u)+D F(x, u)(f(x, u))$ is bounded on some neighborhood of each point $(x, u)$ of Graph(R)

iii) $\forall(x, u) \in \operatorname{Graph}(R), \exists \gamma>0, \exists \varepsilon>0$ such that $\forall(y, v) \in B_{\operatorname{Graph}(R)}((x, u), \varepsilon), \forall z \in B(0, \gamma)$, we have $z \in f_{x}^{\prime}(y, v) f(y, v)+f_{u}^{\prime}(y, v) D F(y, v)(f(y, v))$ $-\mathrm{DT}_{K}(y, f(y, v))(f(y, v))$

We can adapt Theorem 3.2 to viability domains $K$ which are the intersection of a subset $L$ whose tangent cone has a locally compact graph and another subset. Namely, consider the case of a viability domain of the form :

$$
K=L \cap A^{-1}(M)
$$

where $A$ is a $C^{l}$-map from $X$ to a finite dimensional space $Y$. We also assume that

$$
T_{K}(x)=T_{L}(x) \cap T_{A^{-1}(M)}(x) \text { and } T_{A_{(M)}^{-1}}(x)=A^{\prime}(x)^{-1} T_{M}(A x)
$$

This holds true for instance when : 
(3.21) $\left\{\begin{array}{l}L \text { and } M \text { are closed convex subsets and } A \text { is linear, } \\ \text { satisfying } 0 \in \operatorname{Int}(A(L)-M)\end{array}\right.$

(see Aubin-Cellina [1984] p. 325) or when

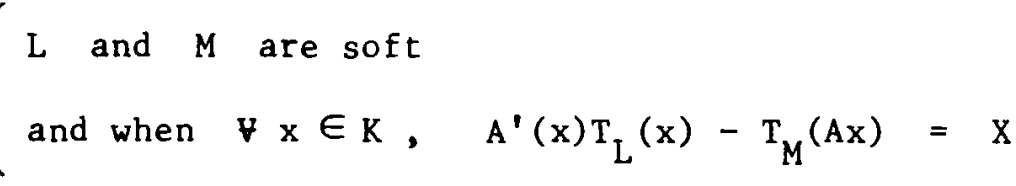

(see Aubin-Ekeland [ 1984 ] p. 440).

In this case, the feedback map $R$ can be written

$$
R(x):=\left\{u \in F(x) \mid f(x, u) \in T_{L}(x) \text { and } A^{\prime}(x) f(x, u) \in T_{M}(A x)\right\}
$$

\section{Corollary 3.3.}

Let us assume that $(3.20)$ holds true and that the graphs of $F$ and $\mathrm{T}_{\mathrm{L}}$ are locally compact. We posit assumptions $(3.7),(3.8),(3.11),(3.12)$ and (3.13) of Theorem 3.2.

Then for any $x_{0} \in K$ and any control $u_{0} \in F\left(x_{0}\right)$ satisfying

$$
A^{\prime}\left(x_{0}\right) f\left(x_{0}, u_{0}\right) \in D_{M}\left(A x_{0}\right)
$$

there exist $T>0$ and a heavy viable trajectory of the controlled system (2.1) on $[0, T]$.

Proof. We replace $F$ by $F \cap T_{L}$, whose graph is locally compact and we observe that $A^{\prime}(x) D_{M}(A x) \subset D_{A^{-1}(M)}(x)$.

Let us formulate Theorem 2 in the particular case of differential inclusions, when $U:=X$ and $f(x, u):=u$. 
Corollary 3.4.

Let us assume that the maps $F$ and $T_{K}$ are soft and satisfy the "transversality condition"

$$
\left\{\begin{array}{l}
v(x, u) \in \operatorname{Graph}(R), v(y, z) \in X \times X, \exists v \in X \text { such that } \\
z \in D F(x, u)(v)-D_{K}(x-u)(v-y)
\end{array}\right.
$$

Then

$$
\mathrm{DR}(\mathrm{x}, \mathrm{u})(\mathrm{v})=\mathrm{DF}(\mathrm{x}, \mathrm{u})(\mathrm{v}) \cap \mathrm{DT}_{\mathrm{K}}(\mathrm{x}, \mathrm{u})(\mathrm{v})
$$

Assume that the graph of $F$ is locally compact and that the regularity condition ( 3.11 ) is satisfied. We posit the first and second order conditions

$$
\left\{\begin{array}{c}
\text { i) } \forall x \in K, \quad R(x):=F(x) \cap T_{K}(x) \neq \emptyset \\
\text { ii) } \quad \forall(x, u) \in \operatorname{Graph}(R), \quad u \in \operatorname{Dom} D F(x, u) \cap \operatorname{Dom}_{\mathrm{DT}}(\mathrm{x}, \mathrm{u})
\end{array}\right.
$$

Then, for any $x_{0} \in K$ and any $u_{0} \in F\left(x_{0}\right)$ satisfying either $u_{0} \in T_{K}\left(x_{0}\right)$ when the graph of $T_{K}$ is locally compact or

$$
u_{0} \in D_{K}\left(x_{0}\right)
$$

then there exist $T>0$ and a $C^{\prime}$ heavy viable trajectory of $x^{\prime} \in F(x)$, $x(0)=x_{0}$ and $x^{\prime}(0)=u_{0}$, a solution to the second order differential equation

$$
x^{\prime \prime}(t)=m\left(\operatorname{DR}\left(x(t), x^{\prime}(t)\right)\left(x^{\prime}(t)\right)\right), \quad x(0)=x_{0}, x^{\prime}(0)=u_{0} .
$$




\section{Proof of the theorem.}

We shall prove this theorem in several steps. We begin by computing the derivative of the feedback map in terms of the derivatives of $f$, $\mathrm{F}$ and $\mathrm{T}_{\mathrm{K}}$.

\section{Lemma 4.1 .}

We posit assumptions (3.7) and (3.8). Then formula (3.9) holds true and $\operatorname{DR}(\mathrm{x}, \mathrm{u})$ is a closed convex process.

Proof.

We set $\phi(x, u):=(x, f(x, u))$ and we observe that

$$
\operatorname{Graph}(R)=\operatorname{Graph} F \cap \phi^{-1}\left(\operatorname{Graph}\left(\mathrm{T}_{\mathrm{K}}\right)\right)
$$

Therefore, we know that

$$
\mathrm{T}_{\operatorname{Graph}(\mathrm{R})}(\mathrm{x}, \mathrm{u}) \subset \mathrm{T}_{\mathrm{Graph}(\mathrm{F})}(\mathrm{x}, \mathrm{u}) \cap \phi^{\prime}(\mathrm{x}, \mathrm{u})^{-1} \mathrm{~T}_{\operatorname{Graph}\left(\mathrm{T}_{\mathrm{K}}\right)}\left(\phi^{\prime}(\mathrm{x}, \mathrm{u})\right)
$$

For proving the other inclusion, we use the formula of Aubin [1983] (see also Aubin-Ekeland, [1984] p. 440) to compute the tangent cone of Graph(R) . The transversality assumption (3.8) implies that

$$
\phi^{\prime}(\mathrm{x}, \mathrm{u}) \mathrm{C}_{\mathrm{Graph}(\mathrm{F})}(\mathrm{x}, \mathrm{u})-\mathrm{C}_{\mathrm{Graph}\left(\mathrm{T}_{\mathrm{K}}\right)}(\mathrm{x}, \mathrm{u})=\mathrm{X} \times \mathrm{X}
$$

Then we deduce that

$$
\mathrm{C}_{\mathrm{Graph}(\mathrm{F})}(\mathrm{x}, \mathrm{u}) \cap \phi^{\prime}(\mathrm{x}, \mathrm{u}) \mathrm{C}_{\mathrm{Graph}\left(\mathrm{T}_{\mathrm{K}}\right)}(\phi(\mathrm{x}, \mathrm{u})) \subset \mathrm{C}_{\mathrm{Graph}(\mathrm{R})}(\mathrm{x}, \mathrm{u})
$$

Since the maps $F$ and $T_{K}$ are soft, then the tangent and contingent cones coincide. Hence inclusions $(4.2),(4.4)$ and $C_{G r a p h(R)}(x, u) \subset T_{G r a p h(R)}(x, u)$ imply the equality 


$$
T_{G r a p h(R)}(x, u)=T_{G r a p h(F)}(x, u) \cap \phi^{\prime}(x, u)^{-1} T_{\operatorname{Graph}\left(T_{K}\right)}(\phi(x, u))
$$

which, obviously, implies formula (3.9). Furthermore, property (2.4)i)

implies that $(x, u) \rightarrow$ Graph $D R(x, u)$ is lower semicontinuous.

We set

$$
G(x, u):=d(0, D R(x, u)(f(x, u))) B
$$

Lemma 4.2.

The trajectories of the first order system of differential inclusions

$$
\left\{\begin{aligned}
\text { i) } & x^{\prime}(t)=f(x(t), u(t)) \\
\text { ii) } u^{\prime}(t) \in G(x(t), u(t)) & (x(0), u(0))=\left(x_{0}, u_{0}\right) \text { given in Graph(R) }
\end{aligned}\right.
$$

which are viable in the sense that

$$
\text { (4.8) } \quad \forall t \geqslant 0, \quad(x(t), u(t)) \in \operatorname{Graph}(R)
$$

are heavy viable trajectories of the controlled system (2.1).

Proof.

Indeed, the viable trajectories of (4.7), (4.8) satisfy

$$
u^{\prime}(t) \in \operatorname{DR}(x(t), u(t))(f(x(t), u(t)))
$$

This inclusion and inclusion (4.7)ii) imply that $u^{\prime}(t)$ belongs to $m(D R(x(t), u(t))(f(x(t), u(t)))$. 
Lemana 4.3.

Let us assume that $f$ is continuous, that the graph of $F$ is locally compact, that

$$
\left\{\begin{array}{l}
(x, u) \in \operatorname{Graph}(R) \rightarrow d(0, \operatorname{DR}(x, u)(f(x, u)) \\
\text { is upper semicontinuous }
\end{array}\right.
$$

and that the first and second order conditions

$$
\left\{\begin{aligned}
& \text { i) } \forall x \in K \quad, \quad R(x) \neq \emptyset \\
& \text { ii) } \forall(x, u) \in \operatorname{Graph}(R), f(x, u) \in \operatorname{Dom} \operatorname{DR}(x, u)
\end{aligned}\right.
$$

hold true.

Then for any $x_{0} \in K$ and any control $u_{0} \in F\left(x_{0}\right)$ satisfying eitier

$$
\mathrm{f}\left(\mathrm{x}_{\mathrm{o}}, \mathrm{u}_{\mathrm{o}}\right) \in \mathrm{T}_{\mathrm{K}}\left(\mathrm{x}_{\mathrm{o}}\right) \text { when } \mathrm{Graph}\left(\mathrm{T}_{\mathrm{K}}\right) \text { is locally compact }
$$

or

$$
f\left(x_{0}, u_{0}\right) \in D_{K}\left(x_{0}\right)
$$

there exist $T>0$ and a heavy viable trajectory of the controlled sysiem $(2,1)$ on $[0, T]$.

\section{Proof.}

a) By Lemma 4.2, we have to prove the existence of viable trajectories to the system $(4.7),(4.8)$. Condition (4.9) is equivalent to the upper semicontinuity of the set valued map $G$. Then

$$
\left\{\begin{array}{l}
(x, u) \rightarrow f(x, u) x G(x, u) \text { is upper semicontinuous with compect } \\
\text { convex values. }
\end{array}\right.
$$

It is also clear that the tangential condition : 
$(4.14)$

$$
(f(x, u) \times G(x, u)) \cap T_{G r a p h(R)}(x, u) \neq \emptyset
$$

is satisfied. If the subset Graph(R) were locally compact, it is sufficient to apply Theorem 2.1 to the problem (4.7)-(4.8) for solving the problem. This is possible under assumption (4.11). Unfortunately, we have observed that this is not necessarily the case. We then shall follow the method proposed by Cornet and Haddad [1983] for solving the viability problem for second order differential inclusions. The idea is to replace Graph $\left(\mathrm{T}_{\mathrm{K}}\right)$ by a locally compact subset $K \subset \operatorname{Graph}\left(\mathrm{T}_{\mathrm{K}}\right)$ large enough for the tangential condition (4.14) to remain valid for $K$. The price to pay is to choose the initial control $u_{0} \in F\left(x_{0}\right)$ such that $f\left(x_{0}, u_{0}\right)$ belongs to the tangent cone $D_{K}\left(x_{0}\right)$ introduced by DubovickiiMiljutin [1963].

b) Assume now that the graph of $T_{K}$ is no longer locally compact and take $f\left(x_{0}, u_{0}\right)$ in $D_{K}\left(x_{0}\right)$.

There exist $\varepsilon>0$ and $\alpha>0$ such that the compact convex subset

$$
\mathrm{k}_{\mathrm{o}}:=\mathrm{x}_{\mathrm{o}}+[0, \alpha]\left(\mathrm{f}\left(\mathrm{x}_{\mathrm{o}}, \mathrm{u}_{\mathrm{o}}\right)+\varepsilon B\right) \subset \mathrm{K}
$$

We observe that the interior of $K_{0}$ is non empty and that $f\left(x_{0}, u_{0}\right)$ belongs to the interior of $\mathrm{T}_{\mathrm{K}_{\mathrm{o}}}\left(\mathrm{x}_{\mathrm{O}}\right)$. Since the graph of $\mathrm{x} \rightarrow \operatorname{Int} \mathrm{T}_{\mathrm{K}_{\mathrm{o}}}(\mathrm{x})$ is open (see Aubin-Cellina [1984] p. 221), there exists $\delta \in] 0, \varepsilon[$ such that

$$
\forall x \in k_{0} \cap\left(x_{0}+\delta B\right), f\left(x_{0}, u_{0}\right)+\delta B \subset \operatorname{Int} T_{K_{0}}(x)=D_{K_{0}}(x)
$$

By the continuity of $f$, there exist $\rho<\delta$ and $n<\delta$ such that :

$$
\begin{aligned}
& \forall x \in k_{0} \cap\left(x_{0}+\rho \dot{B}\right), \forall u \in u_{0}+n \dot{B}, \\
& f(x, u) \in f\left(x_{0}, u_{0}\right)+\delta \dot{B} \subset D_{k_{0}}(x)
\end{aligned}
$$


We set

$(4.17)$

$$
K:=\operatorname{Graph}(F) \cap\left(K_{0} \cap\left(x_{0}+\stackrel{\circ}{\rho}\right) \times\left(u_{0}+\stackrel{\circ}{\eta} \dot{B}\right)\right)
$$

which is obsviously compact.

Since $D_{K_{0}}(x) \subset T_{K_{0}}(x) \subset T_{K}(x)$, we deduce from $(4.16)$ that $K$ is a subset of Graph(R).

c) Let us set $v:=f(x, u)$ and choose $w$ in $m(D R(x, u)(v))$.

So $(v, w)$ belongs to $f(x, u) x G(x, u)$. It is sufficient to check that

$$
\forall(x, u) \in K, \quad(v, w) \in T_{K}(x, u)
$$

for implying the viability condition

$$
\forall(x, u) \in K, \quad f(x, u) x G(x, u)) \cap T_{K}(x, u) \neq \emptyset .
$$

Since $w \in \operatorname{DR}(x, u)(v)$, then $(v, w) \in T_{G r a p h(R)}(x, u) \subset T_{G r a p h}(F)(x, u)$. There exist sequences $h_{n} \rightarrow 0_{+}, v_{n} \rightarrow v$ and $w_{n} \rightarrow w$ such that

$$
\left(x+h_{n} v_{n}, u+h_{n} w_{n}\right) \in \operatorname{Graph}(F)
$$

Also, for $n$ large enough, $x+h_{n} v_{n}$ belongs to $x_{0}+\stackrel{\circ}{B}$ and $u+h_{n} w_{n}$ belongs to $u_{0}+\eta \dot{B}$. Since $f(x, u)$ belongs to $f\left(x_{0}, u_{0}\right)+\delta B$, then $v_{n}$ belongs to $f\left(x_{0}, u_{0}\right)+B$ for $n$ large enough so that, by the very definition of $\mathrm{K}_{0}, \mathrm{x}_{0}+h_{n} v_{n}$ belongs to $\mathrm{K}_{0}$. Hence

$$
\left(x+h_{n} v_{n}, u+h_{n} w_{n}\right) \text { belongs to } K \text { for large } n \text { 's }
$$

and property (4.18) ensues.

d) We then apply the viability theorem : there exists a solutior of the system (4.7) such that, 
$(4.22)$

$$
\text { for } a 11 t \in[0, T], \quad(x(t), u(t)) \in K \subset \operatorname{Graph}(R) \text {. }
$$

This is a heavy solution of the controlled system (2.1).

Proof of Theorem 3.2 .

The first part follows from Lemma 4.1 and the second part from Lemma 4.3 , by observing that (4.9) follows from (3.11).

5. Example: the smooth case.

We consider now the smooth case, when

$$
K:=\{x \in X \mid g(x)=0\}
$$

and when $F$ is the constant map equal to $U$.

Theorem 5.1.

Let us assume that $f$ is a $C^{l}$-function from $X \times U$ to $X$, $g$ is a $C^{2}$-function from $X$ to $Y$ and that, $\forall x \in K, g^{\prime}(x) \in L(X, Y)$ is surjective. We suppose that

$$
\forall x \in K, \quad R(x):=\left\{u \in U \mid g^{\prime}(x) f(x, u)=0\right\} \neq \emptyset
$$

and that

$$
\forall(x, u) \in \operatorname{Graph}(R), \quad g^{\prime}(x) f_{u}^{\prime}(x, u) \in L(U, Y) \text { is surjective. }
$$

Then, for any $x_{0} \in K$ and $u_{0}$ such that $g^{\prime}\left(x_{0}\right) f\left(x_{0}, u_{0}\right)=0$, there exists a heavy viable trajectory, a solution to the system of differential inclusions : 


$$
\begin{aligned}
& \text { i) } x^{\prime}=f(x, u) \\
& \text { ii ) } u^{\prime}=-f_{u}^{\prime}(x, u)^{\star} g^{\prime}(x)^{\star}\left(g^{\prime}(x) f_{u}^{\prime}(x, u) f_{u}^{\prime}(x, u)^{\star} g^{\prime}(x)^{\star}\right)^{-l} \\
& {\left[g^{\prime}(x) f_{x}^{\prime}(x, u) f(x, u)+g^{\prime \prime}(x)(f(x, u), f(x, u))\right]} \\
& \text { iii }(x(0), u(0))=\left(x_{0}, u_{0}\right) \text {. }
\end{aligned}
$$

Proof.

In this simple case, we can compute $m(D R(x, u))(v)$ explicitely. Indeed, since $T_{K}(x)=\operatorname{Ker} g^{\prime}(x)$, then $R(x)=\left\{u \in U \mid g^{\prime}(x) f(x, u)=0\right\}$. By setting :

$$
h(x, u):=\left(g(x), g^{\prime}(x) f(x, u)\right)
$$

we observe that $\operatorname{Graph}(R)=\{(x, u) \mid h(x, u)=0\}$. This function os $c^{\prime}$ and we check easily that

$$
\begin{aligned}
& h^{\prime}(x, u)(v, w)= \\
& \quad\left(g^{\prime}(x) v, g^{\prime \prime}(x)(f(x, u), v)+g^{\prime}(x) f_{x}^{\prime}(x, u) v+g^{\prime}(x) f_{u}^{\prime}(x, u) w\right)
\end{aligned}
$$

Since both $g^{\prime}(x)$ and $g^{\prime}(x) f_{u}^{\prime}(x, u)$ are surjective by assumption, then $h^{\prime}(x, u)$ is surjective. Therefore, this contingent cone to Graph(R) at $(x, u)$ - actually, its tangent space - is the set of pairs $(v, w)$ such that $h^{\prime}(x, u)(v, w)=0$. Hence

$$
\left\{\begin{array}{l}
\operatorname{DR}(x, u)(v)= \\
\quad-f_{u}^{\prime}(x, u)^{-1} g^{\prime}(x)^{-1}\left[g^{\prime \prime}(x)(f(x, u), v)+g^{\prime}(x) f_{x}^{\prime}(x, u) v\right]
\end{array}\right.
$$

Now, we can compute explicitely the element of minimal norm $m(D R(x, u)(v))$ which minimize the norm $\left\|_{w}\right\|$ under the linear constraint 


$$
\left.g^{\prime}(x) f_{u}^{\prime}(x, u) w=-g^{\prime}(x) f_{x}^{\prime}(x, u) v-g^{\prime \prime}(x)(f(x, u), v)\right)
$$

Its solution is given explicitely by the right-hand side of (5.3)ii).

Example. Heavy viable trajectories on affine subspaces.

We consider the case when $K=\{x \in X \mid G x=y\}$ where $G \in L(X, Y)$ is surjective. We assume that

$$
\begin{aligned}
& \text { i) } \forall x \in K, \exists u \in U \text { such that } G f(x, u)=0 \\
& \text { ii) } \forall x \in K, \forall u \in U \text { such that } G f(x, u)=0, G f_{u}^{\prime}(x, u) \\
& \text { is surjective. }
\end{aligned}
$$

Then for any $x_{0}$ satisfying $G x_{0}=y$, $u_{0}$ satisfying $G f\left(x_{0}, u_{0}\right)=0$, there exists a heavy viable trajectory, a solution to the system of differential equations

$$
\left\{\begin{aligned}
\text { i) } \quad x^{\prime} & =f(x, u) \\
\text { ii }) & u^{\prime}=-f_{u}^{\prime}(x, u)^{\star} G^{\star}\left(G f_{u}^{\prime}(x, u) f_{u}^{\prime}(x, u)^{\star} G^{\star}\right)^{-1} G f_{x}^{\prime}(x, u) f(x, u)
\end{aligned}\right.
$$

When $G=g \in x^{\star} \quad(Y=R)$, assumptions (5.6) become

$$
\left\{\begin{array}{l}
\text { i) } \forall x \in K, \exists u \in U \text { such that }\langle g, f(x, u)\rangle=0 \\
\text { ii) } \forall x \in K, \forall u \in U \text { such that }\langle g, f(x, u)\rangle=0, \\
\text { then } f_{u}^{\prime}(x, u)_{g}^{\star} \neq 0
\end{array}\right.
$$

and equation (5.7)ii) becomes

$$
u^{\prime}=-\frac{f_{u}^{\prime}(x, u)^{\star} g}{\left\|f_{u}^{\prime}(x, u)^{\star} g\right\|^{2}}<g, f_{x}^{\prime}(x, u) f(x, u)>
$$


Example. Heavy viable trajectories on the sphere.

Let $G$ be a symmetric positive definite linear operator from $\mathrm{X}$ to $\mathrm{X}^{\star}$ and we take

$$
\mathrm{K}:=\{\mathrm{x} \in \mathrm{X} \mid\langle\mathrm{Gx}, \mathrm{x}\rangle=1\}
$$

We assume that

$$
\left\{\begin{array}{l}
\text { i) } \forall x \in K, \quad R(x):=\{u \in U \mid<G x, f(x, u)>=0\} \neq \emptyset \\
\text { ii) } \forall(x, u) \in \operatorname{Graph}(R), \quad \mathbf{f}_{u}^{\prime}(x, u)^{\star} \mathrm{Gx} \neq 0
\end{array}\right.
$$

Then the heavy viable trajectories on the sphere are the solutions to the system of differential equations

$$
\left\{\begin{aligned}
\text { i) } x^{\prime}= & f(x, u) \\
\text { ii }) u^{\prime}=-\frac{f_{u}^{\prime}(x, u)^{\star} G x}{\left\|f_{u}^{\prime}(x, u)^{\star} G x\right\|^{2}} & (\langle G f(x, u), f(x, u)\rangle \\
& \left.+\left\langle G x, f_{u}^{\prime}(x, u) f(x, u)\right\rangle\right)
\end{aligned}\right.
$$

$\underline{\text { Remark. }}$

Consider the case when

$$
K=\{x \in X \mid g(x)=0\} \cap A^{-1}(M)
$$

where $g$ is a $C^{2}$-map from $X$ to $Y$ and $A$ is a $C^{\prime}$-map from $X$ to $Z, Y$ and $Z$ being finite dimensional spaces. We assume that $y \rightarrow T_{M}(y)$ is lower semicontinuous, that

$$
\forall \mathrm{x} \in \mathrm{K}, \quad \mathrm{A}^{\prime}(\mathrm{x}) \operatorname{Ker} \mathrm{g}^{\prime}(\mathrm{x})-\mathrm{T}_{M}(\mathrm{Ax})=\mathrm{X}
$$

and that, $\forall y, z \in z \times z, \exists v$, a solution to the inclusion : 


$$
\left\{\begin{aligned}
z=D T_{M}\left(A x, A^{\prime} f\right)\left(A^{\prime} v-y\right) & \cap\left[A^{\prime} f_{x}^{\prime} v+A^{\prime \prime}(f, v)\right. \\
& \left.-A^{\prime} g^{\prime-1}\left(g^{\prime \prime}(f, v)+g^{\prime} f_{x}^{\prime} v\right)\right]
\end{aligned}\right.
$$

where we have set $f: f(x, u), g^{\prime}:=g^{\prime}(x), A^{\prime}=A^{\prime}(x), f_{x}^{\prime}=f_{x}^{\prime}(x, u)$, $f_{u}^{\prime}:=f_{u}^{\prime}(x, u), A^{\prime \prime}(u, v)=A^{\prime \prime}(x)(u, v), g^{\prime \prime}(u, v)=g^{\prime \prime}(x)(u, v)$.

Assumption (5.14) implies that

$$
R(x)=\left\{u \in U \mid g^{\prime}(x) f(x, u)=0 \text { and } A^{\prime}(x) f(x, u) \in T_{M}(A x)\right\}
$$

and assumption (5.15) implies that

$$
\left\{\begin{aligned}
\operatorname{DR}(x, u)(v):=-\left(g^{\prime} f_{u}^{\prime}\right)^{-1}\left(g^{\prime \prime}(f, v)+g^{\prime} f_{x}^{\prime} v\right) \cap \\
\left(A^{\prime} f_{u}^{\prime}\right)^{-1}\left(D T_{M}\left(A(x), A^{\prime} f\right)\left(A^{\prime} v\right)-A^{\prime \prime}(f, v)-A^{\prime} f_{x}^{\prime} v\right)
\end{aligned}\right.
$$

Annex.

Let $L \subset X$ and $M \subset Y$ be two closed convex subsets and $A \in L(X, Y)$ satisfying

$$
0 \in \text { Int }(A(L)-M)
$$

Then $K:=\mathrm{L}_{\mathrm{A}}^{-1}(M)$ is a nonempty closed convex subset. Let $\pi_{L}$ and $\pi_{M}$ denote the projectors of best approximation onto $L$ and $M$. Then we can write

$$
m(K)=\pi_{L}\left(-A^{\star} q\right)
$$

where $q$ is a solution to the equation

$$
A \pi_{L}\left(-A^{\star} q\right)=\pi_{M}\left(q+A \pi_{L}\left(-A^{\star} q\right)\right)
$$

Furthermore, $A m(K)=\pi_{M}\left(q+A \pi_{L}\left(-A^{\star} q\right)\right)$. 


\section{REFERENCES}

Aubin, J.P.

[1981] a) Contingent derivatives of set-valued maps and existence of solutions to nonlinear inclusions and differential inclusious. Advances in Mathematics. Supplementary Studies. Ed. L. Nachoin Academic Press. 160-232.

[1981 ] b) A dynamical, pure exchange economy with feedback pricing. J. Economic Behavior and Organizations 2, 95-127.

[1983] Lipschitz behavior of solutions to convex minimization problems. Math. Op. Res.

Aubin, J.P. and A. Cellina

[1984] Differential inclusions. Springer Verlag.

Aubin, J.P. and F.H. Clarke
$[1977]$
Monotone invariant solutions to differential inclusions. J. London

Math. Soc. 16, 357-366.

Aubin, J.P. and I. Ekel and

[1984] Applied Nonlinear Analysis. Wiley Interscience.

Brézis, H.

[1973] Opérateurs maximaux monotones et semi-groupes de contractions dans les espaces de_Hilbert, North-Holland, Amsterdam.

Clarke, F.H.

[1975] Generalized gradients and applications. Trans. A.M.S. 205, 247-262.

[1983] Optimization and nonsmooth analysis. Wiley Interscience.

Cornet, B.

[1981] Contributions à la théorie mathématique des mécanismes dynamiques d'allocation des ressources. Thèse de Doctorat d'Etat. Université de Paris-Dauphine.

Cornet, B. and G. Haddad

[1983] Théorèmes de viabilité pour les inclusions différentielles du second ordre. In Haddad's thesis, Université de Paris-Dauphine.

Dubovickii A.I. and Miljutin A.M.

[1963] Extremum problems with constraints. Soviet Math. 4, 452-455.

Ekeland, I.

[1979] Elements d'économie mathématique; Hermann.

Haddad, G.

[1981] Monotone trajectories of differential inclusions and functional differential inclusions with memory. Israel J. Math. 39, 83-100. 
Penot, J.P.

[1981] A characterization of tangential regularity. J. Nonlinear Analysis T.M.A. 5, 625-643.

Smale, S.

[1976] Exchange processes with price adjustements. J. Math. Econ. $3,211-216$.

Stacchetti, E.

[1983] Analysis of a dynamic, decentralized exchange economy.

Williamson, P.G.

[1985] Palaeontological documentation of speciation in Cenezoic Molluscs from Turkana Basin. Nature. 293, p. 437. 